\title{
More reliable counts of chromosomal aberrations
}

\section{from John R. K. Savage}

A NOVEL application of a staining method widely used to identify newlysynthesised DNA to the problem of obtaining reliable dose-response curves for the effects of radiation damage in cells is reported in this issue of Nature (page 756). The number of chromosomal aberrations induced per cell is the usual measure of radiation exposure. But the precise correlation of dose with the number of chromosomal aberrations it produces is complicated by (amongst other factors) the difficulty of differentiating between cells which have divided a second time after treatment and before analysis. The inclusion of these second division cells in the total cell count inevitably leads to an underestimate of the number of chromosome aberrations produced by any given dose, as the probability of any cell's surviving a second division decreases the larger the number of aberrations it carries. Thus there is selection for less-damaged cells which dilute the scoring sample and reduce the observed yield of aberrations per cell. In a desynchronising cell population like the in vitro blood lymphocyte system, the contamination by second and subsequent division cells will increase progressively with time from initiation and a concomitant fall in the yield of asymmetrical aberrations will be observed.

John R. K. Savage is Head of the Cytogenetics Department, MRC Radiobiology Department, Harwell.
In the past, many workers, aware of this problem, have sampled as early as possible after stimulation, on the principle that the earliest samples are purer first divisions. In practice, a compromise is made to ensure that a sufficient number of mitoses for scoring is present, and for human lymphocytes a time $\sim 48 \mathrm{~h}$ after exposure is usually chosen. Even at this time, second division contamination may not always be negligible and careful workers attempt a further purification of this sample by omitting from their score cells with obviously modified aberrations or those lacking a full complement of acentric fragments.

Now, as Scott and Lyons elegantly demonstrate (page 756) application of the FPG 'harlequin' technique goes a long way towards solving this problem. The technique provides differential staining of the chromatids in second and subsequent division cells based on the semi-conservative segregation of bromodeoxyuridine incorporated in the DNA during synthesis phase. Consequently, by confining scoring to cells with chromosomes not showing differential staining, only first post-trcatment division cells will be included in the sample, irrespective of sampling time. As Scott et al. show, the asymmetrical chromosome-type aberration yield induced in these cells by $200 \mathrm{rad}$ $\mathrm{X}$-rays is constant, independent of sampling time over the range of 40 $72 \mathrm{~h}$. This supplies some evidence for the homogeneous radiosensitivity of the initial population, and provided the constant yield holds for other doses (which seems to be the case from our own preliminary work), the form of dose-response curves obtained with this method should be much more consistent than hitherto. No enhancement of aberration yield was recorded at the concentration of bromodeoxyuridine used or from its method of application. There was, however, a concentration-dependent perturbation of cell progress, but this has little relevance if the primary population is really homogeneous.

The technique is essentially very simple to apply, and there is no reason why it should not be routinely used in all future dose-response curve work. When one considers the tremendous mathematical effort which goes into the fitting and parameter derivation from such curves and their interpretation, it is surely worth-while to ensure that they have a reliable shape before starting.

The advantages of the method are not confined to dose-response curves; one can see many applications in the field of chromosomal aberration studies. For example, in the study of chromosomal mosaics (individuals whose tissues contain mixtures of two or more karyotypically different cell types) the observed relative frequencies of the cell types are in many cases influenced by sclection at cell division. Confining scoring to first division cells may help towards reducing the between-sample variability frequently found.

\section{Are protons unstable?}

from F. E. Close

THE experimental lower limit on the proton lifetime is well over a trillion times the age of the Universe and this has led people to believe that the proton is indeed absolutely stable. Recent theoretical attempts to construct theories that unify the strong force with the weak-electromagnetic force (see Nature 277, 349; 1979), however, have the consequence that the proton is not absolutely stable. A lifetime as 'short' as $10^{31}$ years could be possible (about an order of magnitude greater than the present lower limit).

Quantum electrodynamics (QED) and quantum chromodynamics (QCD) (the theory of the strong interaction) are very similar field theories and appear ir many ways to be profoundly and intimately related. Recently devel- oped ideas on grand unification of the natural forces do just this and postulate that they are related in a way that is made precise by group theory. Mathematically, QED is a $U(1)$ field theory and QCD is an $\mathrm{SU}(3)$ ("non Abelian") field theory, the U(1) essentially displaying the fact that there is but one kind of electrical charge (which can be positive or negative like the set of real numbers) and the SU(3) displaying the existence of three kinds of colour charge (often called red, yellow and blue by analogy with the primary colours). In a nutshell, QCD is like QED with $3 \times 3$ matrices in place of real numbers in the 'photon' (coloured gluon) couplings. (Photons are the quanta of the electromagnetic force: coloured gluons those of the strong interaction.) To unify these, find a group $\mathrm{G}$ which contains $\mathrm{SU}(3) \times \mathrm{U}(1)$

F. E. Close is at the Rutherford Laboratory.

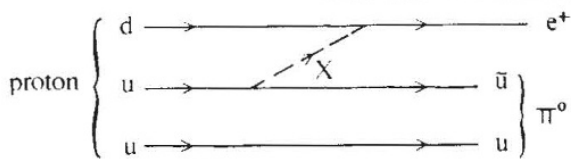

a possible source of proton decay

and postulate that there exists a corresponding field theory. The SU(3) QCD and U(1) QE:D will then be two particular subclasses of this grand field theory. Unification of QCD with the electromagnetic and weak interaction can be achieved if $G$ is the group SU(5) or $\mathrm{SO}(10)$. A consequence of this unification is that at energies typical of the world about us the SU(3) colour force is stronger than the $U(1)$ electromagnetic. This is in accord with observed atomic structure: the protons and neutrons (quark systems) are bound in the nucleus by the strong force while the electron (lepton) orbits the nucleus at large distances held by the electromagnetic force.

An outcome of such a unification is that in addition to the photon and the 